\title{
A novel method of combining Periodic Acid Schiff staining with Wright-Giemsa staining to identify the pathogens Penicillium marneffei, Histoplasma capsulatum, Mucor and Leishmania donovani in bone marrow smears
}

\author{
LINGYAN QIN $^{1}$, LIGANG ZHAO $^{1}$, CHUNYAN TAN $^{2}$, XU CHEN $^{1}$, ZHENG YANG $^{1}$ and WUNING MO \\ ${ }^{1}$ Department of Clinical Laboratory, First Affiliated Hospital of Guangxi Medical University; \\ ${ }^{2}$ Department of Clinical Laboratory, People's Hospital of Guangxi Zhuang Autonomous Region, \\ Nanning, Guangxi 530021, P.R. China
}

Received May 23, 2014; Accepted January 29, 2015

DOI: $10.3892 /$ etm.2015.2357

\begin{abstract}
Penicillium marneffei, Histoplasma capsulatum, Mucor and Leishmania donovani can lead to penicilliosis marneffei, histoplasmosis, mucormycosis and leishmaniasis, respectively, which, to a certain extent, share similar clinical manifestations. These pathogens are approximately the same size, therefore it is relatively difficult to rapidly diagnose the diseases. The aim of the present study was to explore a novel method that attempts to rapidly identify the pathogens of these diseases. In the Wright-Giemsa staining, the four pathogens were approximately the same size and mainly existed in macrophages. The multiplying $P$. marneffei had two nuclei, which were on both sides of the fungus, and had light cross-walls in the middle. H. capsulatum had a purplish nucleus, which occupied between one-third and one-half of the spore. The cytoplasm was light blue. Peripheral spores were observed in the form of an empty, bright ring without color, like a capsule. Generally, Mucor were observed to have a long and lightly stained area, which could be easily confused with the Wright staining of dinuclear P. marneffei. L. donovani exhibited a deep-staining kinetoplast near the nucleus. In the Periodic Acid Schiff (PAS) staining, the pathogens of P.marneffei and H. capsulatum were distinct and stained red. Differentiation between $P$. marneffei and $H$. capsulatum relied on their modes of reproduction:
\end{abstract}

Correspondence to: Mr. Zheng Yang or Professor Wuning Mo, Department of Clinical Laboratory, First Affiliated Hospital of Guangxi Medical University, 6 Shuangyong Road, Nanning, Guangxi 530021, P.R. China

E-mail: jackyyoung@foxmail.com

E-mail: mown16300@126.com

Key words: Penicillium marneffei, Histoplasma capsulatum, Mucor, Leishmania donovani, bone marrow smear, Wright-Giemsa staining, Periodic Acid Schiff staining
P. marneffei depends on fission, when the pathogens stretch into sausage-shapes and are split by a cross-wall, while $H$. capsulatum depends on budding so that narrow-necked, single spores can be formed. With PAS staining, the cell walls and intracellular contents of Mucor and L. donovani were not stained, lightly stained or granulated and discontinuous. In conclusion, this method, combining PAS and Wright-Giemsa staining, is simple and rapid, and may contribute to the effective identification of the four pathogens.

\section{Introduction}

Penicillium marneffei is an opportunistic pathogenic fungus and the only thermally dimorphic species in the Penicillium genus (1). The pathogen can lead to disseminated infection in immunocompromised patients, particularly those infected with human immunodeficiency virus (HIV). Penicilliosis marneffei has become one of the most common diseases in patients with acquired immune deficiency syndrome (AIDS) in Southeast Asian nations, such as Southern China, Thailand, Malaysia, the Philippines, Vietnam and Singapore. If penicilliosis marneffei is not diagnosed early and treated appropriately, its mortality rates can reach $91.3 \%$ (2).

Histoplasma capsulatum can lead to histoplasmosis, a systemic mycosis affecting humans and animals that is considered to be a leading indicator of HIV infection. Histoplasmosis was previously a rare disease; however, since the 1980s it has developed into a major opportunistic infection in patients with AIDS. Although the majority of patients with histoplasmosis only present with mild symptoms, $\sim 5 \%$ are likely to develop a severe and life-threatening disease if early diagnosis is not conducted $(3,4)$.

Mucor, a serious opportunistic pathogenic fungus, can cause mucormycosis. Patients with immunosuppression or diabetes are more sensitive to the acquisition of mucormycosis (5). Mucormycosis is a dangerous illness with a high mortality rate (6).

Leishmania donovani causes visceral leishmaniasis, a zoonosis and one of most important emerging diseases 
worldwide. Its wide distribution and global prevalence lead to serious consequences; at present the condition is prevalent in 88 countries, affecting $\leq 1.2$ billion individuals ( 7 ).

The described diseases are seriously harmful to human health and have a significant impact on the life conditions of the affected individuals. Pathogen culture is the most reliable method of identifying the pathogen, but this takes a long time. The rapid identification of the four pathogens and the early diagnosis of the four diseases are therefore of great importance; however, each of the pathogens have a similar size and shape in the Wright-Giemsa staining of bone marrow smears (BMSs). Furthermore, the clinical manifestations of the four diseases are similar, to a certain extent, since the four pathogens always invade the mononuclear phagocyte system and can be found in the macrophages. The aim of the present study was therefore to explore a novel method combining Periodic Acid Schiff (PAS) and Wright-Giemsa staining for BMSs in order to rapidly identify and distinguish the four pathogens.

\section{Materials and methods}

Specimens. The BMSs ( $\mathrm{n}=55)$ were obtained from bone marrow invaded by P. marneffei (25 BMSs), H. capsulatum (10 BMSs), Mucor (3 BMSs) and L. donovani (17 BMSs). The diagnosis of the four diseases (penicilliosis marneffei, histoplasmosis, mucormycosis and visceral leishmaniasis) was confirmed by pathogen culture. The cases of penicilliosis marneffei and visceral leishmaniasis were from the First Affiliated Hospital of Guangxi Medical University (Nanning, China); the cases of mucormycosis were from the People's Hospital of Guangxi Zhuang Autonomous Region (Nanning, China); and the cases of histoplasmosis were referred by experts from other hospitals and identified and confirmed by the Shanghai Huashan Hospital (Shanghai, China) and Nanjing Institute of Skin Disease (Nanjing, China). Informed consent was obtained from all patients prior to participation in the study and ethical approval was obtained from the First Affiliated Hospital of Guangxi Medical University Ethical Review Committee (Nanning, China).

PAS staining methods. Each BMS was fixed by $95 \%$ alcohol for $10 \mathrm{~min}$, prior to being washed, dried, stained by $1 \%$ periodic acid for $20 \mathrm{~min}$ and washed and dried once more. Each BMS was then stained by a Schiff reagent for $60 \mathrm{~min}$, washed and dried, and subsequently stained by hematoxylin for $5 \mathrm{~min}$, washed and dried. The BMSs were observed using light microscopy. Wright-Giemsa staining was also performed, following standard protocols.

\section{Results}

P. marneffei. The pathogens were mostly located in the cytoplasm of macrophages; a few pathogens were freely scattered extracellularly. In the Wright-Giemsa staining, the yeast forms of P. marneffei were round, ellipsoidal or sausage-like with one or two purplish-red, small nuclei and a light blue cytoplasm. The multiplying $P$. marneffe $i$ had two nuclei, which were on both sides of the fungus. The P. marneffei pathogens were not of a uniform size and measured 2-8 $\mu \mathrm{m}$.
The cell wall was not clearly visible (Fig. 1A). In the PAS staining, the cell walls of the P. marneffei were red, distinct and continuous. A number of sausage-like cells with cross-walls could be observed and the intracellular contents did not stain easily (Fig. 1B).

$H$. capsulatum. In the Wright-Giemsa staining, the majority of the histoplasmosis-causing pathogens were located in the cytoplasm of macrophages; only a few pathogens were observed to be outside of the macrophages. The H. capsulatum exhibited round or ellipsoidal shapes and measured 2-5 $\mu \mathrm{m}$. The cell membrane of the pathogens was not clearly visible. A purplish nucleus, which occupied between one-third and one-half of the spore, and a light blue cytoplasm could be observed in the pathogens. Peripheral spores formed a halo-like circle (Fig. 2A). Differentiation between the $H$. capsulatum and $P$. marneffe $i$ was difficult under Wright-Giemsa staining; however, significant differences between $H$. capsulatum and P. marneffe $i$ were observed with PAS staining. The cell walls of the H. capsulatum were red, distinct and continuous, and the intracellular contents were not easily stained. No sausage-like cells or cross-walls were visible but, occasionally, narrow-necked, single spores could be observed (Fig. 2B). It was not difficult, therefore, to identify the two pathogens when using Wright-Giemsa staining combined with PAS staining.

Mucor. In the Wright-Giemsa staining, the location of the Mucor pathogens was similar to that of the P. marneffei and H. capsulatum pathogens. With the exception of a few pathogens outside the macrophages, others were located in the macrophage cytoplasm. The majority of the pathogens were round and a few were ellipsoidal; no sausage-like pathogens were observed. The size of the pathogens varied between 2 and $5 \mu \mathrm{m}$. The cell membrane of the pathogens could not be clearly observed. A purplish-red, small nucleus and light blue cytoplasm were evident in the pathogens. The boundary of the pathogens was indistinct. A long, lightly stained area could be noted in the majority of pathogens, which made the cell body appear to have two separate, half-moon-shaped areas. This was easily confused with the dinuclear P. marneffei (Fig. 3A). In the PAS staining, the cell walls of the pathogens were not stained, lightly stained or granulated and discontinuous, which made it easier to differentiate Mucor from P. marneffei and H. capsulatum (Fig. 3B).

L. donovani. In the Wright-Giemsa staining, the majority of the L. donovani pathogens were located in the cytoplasm of the macrophages, and only a few pathogens were located extracellularly. The majority of the pathogens exhibited round or ellipsoidal shapes; only a few were sausage-like. Their sizes varied between 2 and $5 \mu \mathrm{m}$. The boundary of the pathogens could not be clearly observed. A purplish-red, small nucleus and light blue cytoplasm were evident in the pathogens. Furthermore, the L. donovani exhibited a thin, rhabditiform or granulated, purplish kinetoplast near the nucleus; this should not be confused with the dual-core P. marneffei (Fig. 4A). In the PAS staining, the cell walls and intracellular contents of the pathogens were lightly stained or not stained. It was therefore relatively simple to identify $L$. donovani when combining PAS and Wright-Giemsa staining (Fig. 4B). 


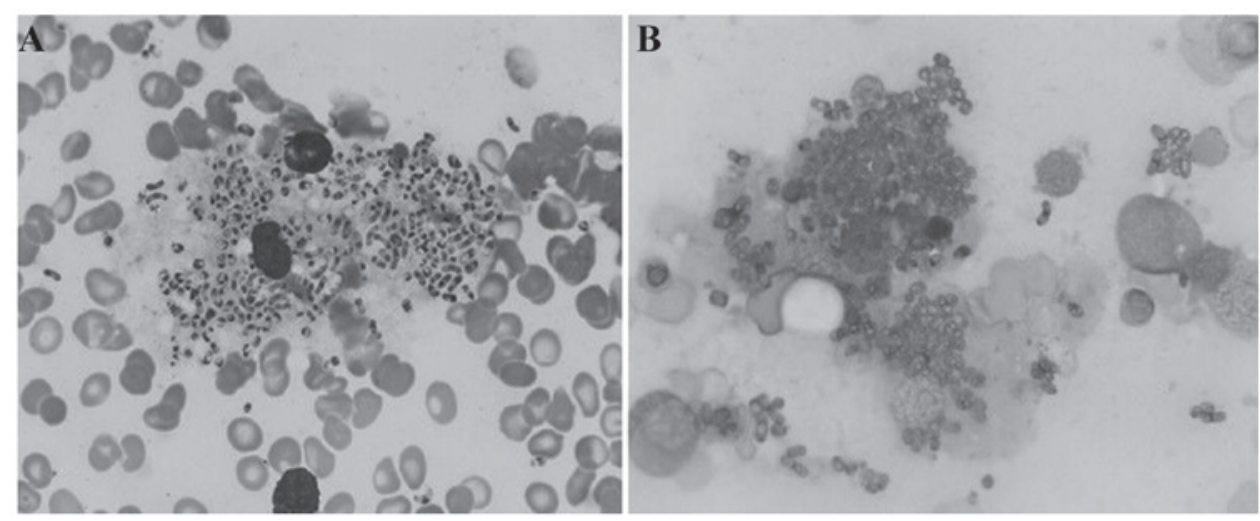

Figure 1. Staining of Penicillium marneffei. (A) The P. marneffei pathogens are tightly packed in the macrophages. Sausage-like cells and cross-walls can be observed (Wright-Giemsa staining; original magnification, 10x100). (B) Staining shows the sausage-like cells and cross-walls. In the central field the P. marneffei pathogens packed together are engulfed inside the cytoplasm of macrophages (Periodic Acid Sciff staining; original magnification, 10x100).
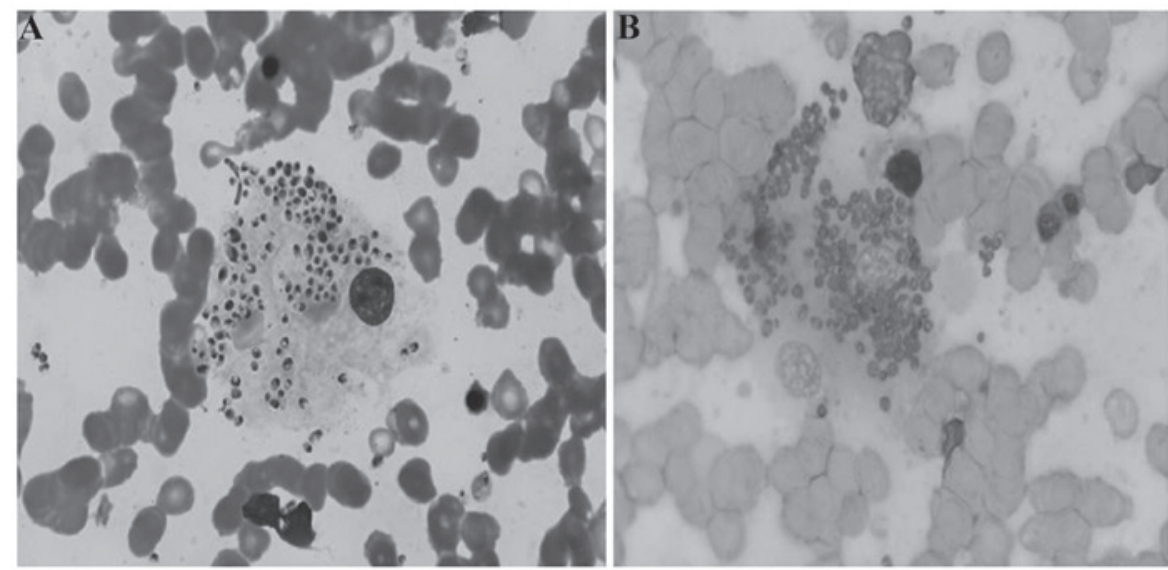

Figure 2. Staining of Histoplasma capsulatum. (A) The H. capsulatum pathogens are tightly packed in the macrophages. Peripheral spores formed a halo-like circle (Wright-Giemsa staining; original magnification, 10x100). (B) The intracellular contents are not clearly stained; no sausage-like cells or cross-walls can be observed. In the central field the pathogens of $H$. capsulatum are engulfed inside the cytoplasm of macrophages (Periodic Acid Schiff staining; original magnification, 10x100).
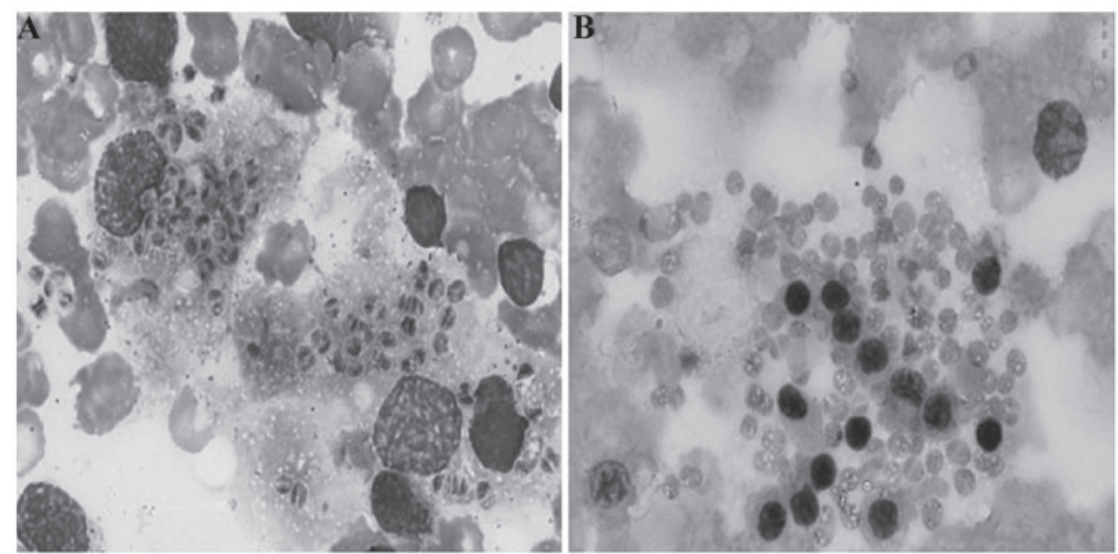

Figure 3. Staining of Mucor. (A) The Mucor pathogens are tightly packed in the macrophages. A long, lightly stained area can be observed in the majority of the pathogens, making the cell body appear to have two separate, half-moon-shaped areas (Wright-Giemsa staining; original magnification, 10x100). (B) The cell walls of the pathogens are not stained, lightly stained or granulated and discontinuous (Periodic Acid Schiff staining; original magnification, 10x100).

\section{Discussion}

Penicilliosis marneffei, histoplasmosis, mucormycosis and visceral leishmaniasis are commonly found in immunocompromised patients (1). In the present study, a total of 55 patients who suffered from the four described diseases were enrolled. It was found that all the patients were HIV-positive, with the exception of one patient with mucormycosis, who 


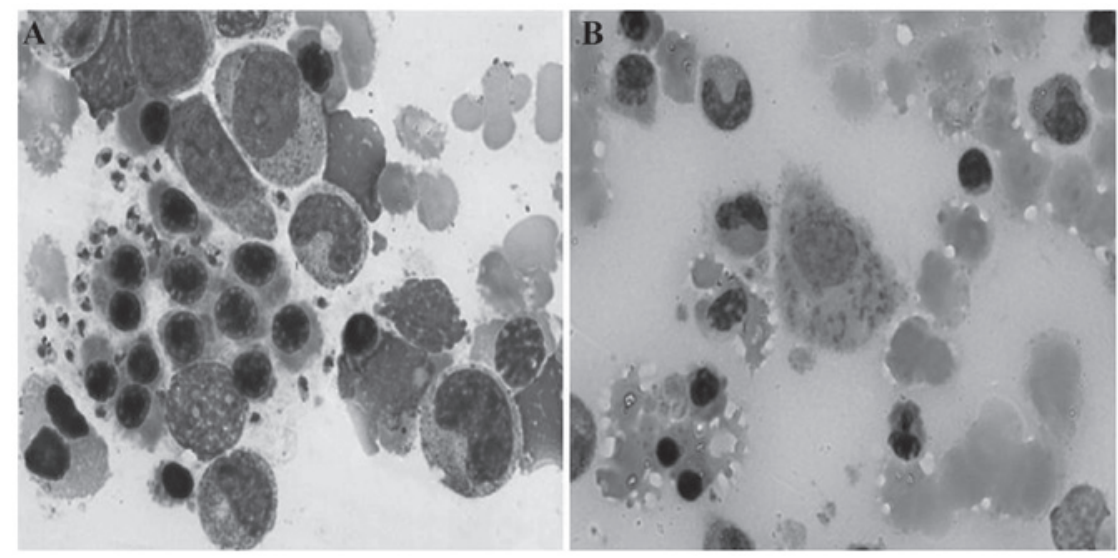

Figure 4. Staining of Leishmania donovani. (A) The L. donovani pathogens are tightly packed in the macrophages. A kinetonucleus, which is thin and rhabditiform or granulated and purplish can be observed (Wright-Giemsa staining; original magnification, 10x100). (B) The cell walls and intracellular contents of the pathogens are lightly stained or not stained. (Periodic Acid Schiff staining; original magnification, 10x100).

suffered from disseminated intravascular coagulation caused by multiple organ damage; this patient succumbed having only been in hospital for two days, meaning that the HIV test had not yet been performed. The clinical manifestations, including fever, hepatosplenomegaly, anemia and emaciation, were evident in each of the 55 patients. The size and shape of the four pathogens on the BMSs were similar, making it relatively difficult to discriminate between them based solely on Wright-Giemsa staining.

In the present study, a novel method of combining Wright-Giemsa staining with PAS staining was utilized to help to solve this problem. To the best of our knowledge, this is the first study to provide a new method to identify the four easily confused pathogens with Wright-Giemsa staining. P. marneffei and H. capsulatum can cause serious systemic infections. Following staining with PAS, the cell walls of these pathogens were red and their outlines were clear and distinct. It was therefore possible to observe and distinguish the two pathogens under a low-power lens. Furthermore, as P. marneffei depends on fissiparity, cells with cross-walls could be observed in the mitotic phase. Cross-walls are the cell walls of two unseparated cells, and they therefore appear thick and deep red in PAS staining. The characteristic septate forms and the absence of budding enable the differentiation between P. marneffei and H. capsulatum. We propose that fission is a reliable indicator for the diagnosis of penicilliosis marneffei $(8,9)$.

H. capsulatum and Mucor depend on spore reproduction. Neither sausage-like cells nor cross-walls appeared in the two pathogens, but narrow-necked, single spores could be observed in microscopy examination of H. capsulatum. A long, lightly colored area was evident in the majority of the Mucor pathogens, which made the cell body appear in the form of two separate, half-moon-shaped areas. In the PAS staining, the cell walls of the pathogens were not stained, lightly stained or granulated and discontinuous, which made it easier to distinguish Mucor from P. marneffei and H. capsulatum.

L. donovani can cause visceral leishmaniasis in humans, dogs and wild animals. In the Wright-Giemsa staining, the pathogens exhibited a purplish-red, small nucleus and a thin, rhabditiform or granulated, purplish kinetoplast. In the
PAS staining, the cell walls and intracellular contents of the pathogens were lightly stained or not stained. It was therefore relatively simple to distinguish $L$. donovani from the dual-core P. marneffei when combining PAS and Wright-Giemsa staining.

P. marneffei, H. capsulatum and Mucor are opportunistic pathogens. While microbiological culture remains the 'gold standard' for the diagnosis of opportunistic infection, the examination takes 3-10 days. Examinations of biopsied tissue samples provide an early diagnosis, as the fungus can be identified by the representative morphological characteristics. The examination of BMSs is always conducted for morphological examination in patients with AIDS, and the cases in the present study were also patients with HIV infection. The examination of BMSs lays the foundation for the further identification and early diagnosis of the four pathogens and their corresponding diseases.

In conclusion, the four diseases of the present study can lead to similar clinical manifestations, which include the first symptoms of headache, cough or fever. Furthermore, these pathogens are found in patients with low immunity and exhibit similarities in microscopic morphology and size. They are additionally all located in mononuclear macrophages, meaning that the four pathogens are easily confused. The differentiation of the four pathogens is therefore relatively problematic when relying solely on Wright-Giemsa staining. In the present study, it was shown that a method combining PAS and Wright-Giemsa staining could effectively enhance the identification of the four pathogens. This method is simple and can enable the rapid diagnosis of penicilliosis marneffei, histoplasmosis, mucormycosis and visceral leishmaniasis. Furthermore, the method may have an important role in diagnosing the four diseases in cases in which it is not possible to conduct pathogen culture and serology tests.

\section{References}

1. CooperCRJrandMcGinnis MR:Pathology of Penicilliummarneffei. An emerging acquired immunodeficiency syndrome-related pathogen. Arch Pathol Lab Med 121: 798-804, 1997.

2. Bo L and Ping F: Research progress of penicilliosis marneffei. J Dermatol Venereol 32: 26-28, 2010 (In Chinese). 
3. Freifeld AG, Iwen PC, Lesiak BL, Gilroy RK, Stevens RB and Kalil AC: Histoplasmosis in solid organ transplant recipients at a large Midwestern university transplant center. Transpl Infect Dis 7: 109-115, 2005.

4. Kauffman CA: Histoplasmosis: a clinical and laboratory update. Clin Microbiol Rev 20: 115-132, 2007.

5. Kauffman CA and Malani AN: Zygomycosis: an emerging fungal infection with new options for management. Curr Infect Dis Rep 9: 435-440, 2007.

6. Roden MM,Zaoutis TE, Buchanan WL, et al: Epidemiology and outcome of zygomycosis: a review of 929 reported cases. Clin Infect Dis 41: 634-653, 2005.
7. Singh S and Sivakumar R: Challenges and new discoveries in the treatment of leishmaniasis. J Infect Chemother 10: 307-315, 2004.

8. Mo W, Deng Z and Li S: Clinical blood routine and bone marrow smear manifestations of disseminated penicilliosis marneffei. Chin Med J (Engl) 115: 1892-1894, 2002.

9. Deng Z and Liu X: Disseminated Penicilliosis marneffei in a patient with acquired immunodeficiency syndrome: a first case report from China. Chin Med J (Engl) 113: 1049-1050, 2000 . 